\title{
COMMUNICATION
}

\section{Détection précoce d'un carcinome épidermoïde chez une patiente atteinte de la maladie de Fanconi}

\section{Maffi-Berthier $\mathrm{L}^{1}$, Renoux $\mathrm{M}^{2}$, Ejeil $\mathrm{AL}^{3}$}

1 - Interne de médecine bucco-dentaire à l'université Paris V, AP-HP - Hôpitaux Universitaires Paris Nord Val de Seine, Service d'Odontologie de l'hôpital Bretonneau, Paris

2 - AHU à l'Université Paris V, AP-HP - Hôpitaux Universitaires Paris Nord Val de Seine, Service d'Odontologie de I'hôpital Louis Mourier, Colombes

3 - MCU-PH à l'université Paris V, AP-HP - Hôpitaux Universitaires Paris Nord Val de Seine, Service d'Odontologie de l'hôpital Bretonneau, Paris

\section{Introduction}

L'anémie de Fanconi ou maladie de Fanconi est une affection génétique rare avec une fréquence estimée à 1/350.000 naissances en France. Elle est due à différentes mutations de gènes impliqués dans le système de réparation de l'ADN. Ces mutations génèrent une grande hétérogénéité de phénotypes pathologiques bien que certaines manifestations cliniques soient communes à une majorité des patients, telle la propension à développer des cancers de la cavité buccale et de la sphère oropharyngée.

\section{Observations}

Une patiente âgée de 57 ans se présente à la consultation de pathologie des muqueuses buccales de l'hôpital Bretonneau sur le conseil d'un confrère parodontiste. Elle se sait atteinte d'une maladie de Fanconi diagnostiquée depuis une dizaine d'années.

On note dans ces antécédents médicaux une anémie idiopathique ancienne sans étiologie, des transaminases élevées, une formule sanguine symptomatique (hémoglobine basse, taux plaquettaire faible, leucopénie) un antécédent de polype cancéreux dans l'œsophage au niveau de l'aryténoïde gauche traité par chirurgie et radiothérapie en 1994, une ostéoporose et une ménopause précoce.

Elle est traité par Aranesp 500 (1 injection par mois), Seroplex $5 \mathrm{mg}$ (1 comprimé par jour) et Uvedose (1 ampoule par mois). Elle est traitée depuis environ 4 ans par son dentiste pour une gingivite récidivante en secteur 4 et évoque notamment des saignements et des œdèmes gingivaux fréquents.

L'examen clinique révèle une lésion érythroleucoplasique et ulcérée en vestibulaire de 44,45 et 46, non douloureuse, sans autres lésions sur les muqueuses voisines.

Du fait d'une thrombopénie importante, la patiente est orientée vers l'institut Curie pour une biopsie. Le résultat anatomopathologique met en évidence la présence d'un carcinome in situ. Un bilan d'extension est alors entrepris et une exérèse carcinologique est réalisée. 


\section{Discussion}

La maladie de Fanconi résulte d'un processus de non réparation des lésions acquises de l'ADN. II en résulte des anomalies hématologiques constantes qui peuvent être compensées, mais aucune thérapie actuelle ne permet de traiter l'étiologie de la pathologie. Les patients atteints de la maladie de Fanconi restent donc définitivement à risque élevé de développer un carcinome. Ce risque augmente avec l'âge et l'augmentation naturelle des " erreurs » lors de la réplication de l'ADN. Les carcinomes épidermoïdes de la sphère oro-faciale et de la vulve sont les plus fréquents du fait de leur exposition constante aux agressions extérieures et des propriétés de leurs composants tissulaires.

\section{Conclusion}

Un examen annuel des patients atteints de la maladie de Fanconi doit être systématique à la recherche de lésions suspectes ou à risque de transformation. Certains auteurs préconisent une auto-inspection des patients afin qu'ils consultent rapidement.

\section{Références}

Rosenberg P.S. and al. Cancer incidence in persons with Fanconi Anemia. Blood 2003;101:822-6.

Rosenberg P.S. et al. Risk of head and neck squamous cell cancer and death in patients who did and did not receive transplants. Blood 2005;105:67-73.

Alter B.P and al. "Cancer in Fanconi Anemia, 1927-2001." Cancer 97, no. 2 (January 15,2003): 425-40. 